\title{
Role of neurotransmitters in schizophrenia: a comprehensive study
}

\author{
Videsha Bansal ${ }^{1}$, Indranath Chatterjee ${ }^{2, *}$ \\ ${ }^{1}$ Dept. of Chemical Engineering, Institute of Engineering and Technology, JK Lakshmipat \\ University, Jaipur - 302026, India \\ ${ }^{2}$ Dept. of Computer Engineering, Tongmyong University, Busan - 48520, South Korea \\ *Corresponding author: indranath.cs.du@gmail.com
}

\begin{abstract}
Schizophrenia is a psychological disorder, way tougher to diagnose than other psychological disorders, as it shares similar symptoms with psychosis. As we know, schizophrenia occurs due to chemical imbalance in the brain; identifying the role of neurotransmitters in schizophrenia poses a vital area to study. Neurotransmitters being the sole carrier of different brain activities, researchers have already initiated studies to investigate their role and effect in disorder. Firstly, this paper performs a critical review of the literature that dealt with different neurotransmitters in schizophrenia. Secondly, we identify the most important neurotransmitters and broadly elaborate on their functional roles and effects on the disorder. Finally, we have successfully identified various gaps and unexplored research questions to investigate these neurochemicals' role. Studies show that neurotransmitters like dopamine, glutamate, GABA, serotonin, and oxytocin are majorly responsible for schizophrenia, among which dopamine contributes the most. To the best of our knowledge, this paper encapsulates all the neurotransmitters, enzymes, and chemicals for the first time and explores their related literature. This study also identifies the most responsible chemicals involved in schizophrenia and unfolds the research community's unsolved problem.
\end{abstract}

Keywords: Brain hormones; neurochemistry; neurotransmitter; psychological disorder; schizophrenia.

\section{Introduction}

Schizophrenia is a severe mental disorder that clinicians encounter and is affecting millions of people around the globe. It is a chronic and disabling mental illness (Chatterjee et al., 2018a; Chatterjee \& Mittal, 2019). The sufferers face different positive, negative, and cognitive symptoms like hallucination, delusion, disorganization, difficulty in emotional expressions, and lack of motivation and attention (Gupta \& Kulhara, 2010; Swanton, 2020).
The three unavoidable clinical facts on schizophrenia as stated by Weinberger. (1987) are: first, there is a very high probability that the clinical manifestation of the disorder will occur during the end of adolescence or early adulthood; second, the role of stress during the onset and relapse of the disorder; and third, the therapeutic efficacy of neuroleptic drugs (Weinberger, 1987). Recent studies of neurodevelopmental mechanisms strongly suggest that no single gene or factor is 
responsible for driving a highly complex biological process (Lewis \& Levitt, 2002). When neurodevelopment and schizophrenia are considered together, there exist various parameters, which needs to be acknowledged, such as:

- It is a disorder of the brain maturation cortex (cerebral). The disorder becomes active in the early days rather than progressing parallelly to its clinical progression (Lewis \& Levitt, 2002).

- Pathologically, schizophrenia is not neurodegenerative (Lewis \& Levitt, 2002).

Although the approaches have changed, studies of the etiology of schizophrenia are still in progress. Due to this disorder, many factors have been reported, from psychotherapy to autoimmune to genetic factors. Studies discuss the relationship between age and the healthy human brain's development (Gupta \& Kulhara, 2010; Chatterjee et al., 2020b). There are some essential facts about healthy neurological development, i.e., firstly, different brain areas are produced or developed at different times. Therefore, the time spends by the growing embryo in intrauterine life is a significant determination of later discrepancy. Secondly, minor anomalies in the initial stages can produce substantial differences in the healthy human brain's development. Finally, a set of specific molecular signals are responsible for specific roles at various stages of neurodevelopment. Researchers suggest three aspects of the brain, which are developed one by one, i.e., working memory (Chatterjee et al., 2019), cognitive control, and reasoning, which keep evolving with ages (Catts et al., 2013).
In 2018, Raine. (2018) stated that neurodevelopment disorder has its origin in childhood, which is characterized by structural and functional abnormalities in the brain. It is often accompanied by neurocognitive impairments leading to disturbed social and academic behaviors. The course of schizophrenia may be divided into four stages: interference by neurotransmitters (Insel, 2010).

- Stage I - features of this stage are the genetic vulnerability and the environmental risk, which could be diagnosed by family background.

- $\quad$ Stage II - a feature of this stage is help seeking, which could be diagnosed by cognitive assessment.

- Stage III - This stage's feature is behavior recurrence, which could be diagnosed by clinical interview.

- $\quad$ Stage IV - This stage's feature is losses of functions; which clinical interviews could diagnose.

As we know, neurotransmitters play a vital role in neurodevelopmental disorders. According to the Oxford dictionary, the definition of neurotransmitter is stated as "a substance that is released at the end of the nerve fiber by the arrival of a nerve impulse and spreading through the synapse or junction transfers the impulse to another nerve fiber" (Dictionary, 1989). The neurotransmitters are classified into different chemical groups according to their role, summarized in Table 1.

As schizophrenia is induced by different neurotransmitters and several other factors 
such as social, environmental, genetic, and drug-related reasons, as shown in Figure 1, various types exist of this disorder. Research (Geisler et al., 2015; Chatterjee \& Mittal, 2019) shows five types of schizophrenia. They can be differentiated concerning the symptoms.

(i) Paranoid schizophrenia - It is characterized by paranoid behavior that includes delusions and auditory hallucinations (Chatterjee, 2018b). Paranoid behaviors manifest themselves through feelings of persecution, surveillance, and delusion of becoming a famous person. People with paranoid schizophrenia sometimes express anger, anxiety, and hostility. The person usually has a relatively normal intellectual functioning (Zhou et al., 2007).

(ii) Disorganized schizophrenia - A person with disorganized schizophrenia will manifest disordered behaviors or speech that become difficult to understand. The patients with disorganized schizophrenia show inappropriate emotions that may not relate to the present situation. Daily lifestyle may also get hampered, including hygiene, food, and work due to their negligence and disorganized thinking patterns (Brune, 2003).

(iii) Catatonic schizophrenia - Movement disorders are characterized by catatonic schizophrenia. Patients suffering from this type of schizophrenia can show extreme symptoms such as remain motionless for hours or may move in all directions. They may remain silent or may talk for hours. These behaviors expose people with catatonic schizophrenia to high risk since they become unable to take care of themselves (Beckmann et al., 1996). (iv) Undifferentiated schizophrenia - This type of disorder is seen when a person shows mixed behaviors that include at least two other types of schizophrenia. This type of disorder symptoms includes delusions, hallucinations, speech or disorganized behavior, catatonic behavior (Kendler et al., 1985).

(v) Residual schizophrenia - When a person shows no symptoms of schizophrenia, despite having at least one episode of the disorder earlier in his life, the person may be in full remission or some time may resume symptoms (Tang et al., 1994).

A report $(2019)^{1}$ by the World Health Organization states that schizophrenia affects more than 23 million people worldwide but is not as common as many other mental disorders. It is more common in males (12 million) than females (9 million) and also strikes before in men than women.

This paper explores the various neurochemical aspects of schizophrenia, starting from its definition to multiple types of schizophrenia, stage-wise symptoms, and all essential neurotransmitters related to this disorder. Firstly, we have reviewed the various researches carried on the involvement of neurotransmitters in schizophrenia. Secondly, we discuss all the neurotransmitters responsible for schizophrenia. Thirdly, we critically investigate each neurotransmitter's role in various characteristics of the disorder and explore the interconnection between these neurotransmitters. Finally, this study finds quite a few further research opportunities

\footnotetext{
${ }^{1}$ Schizophrenia. (n.d.). Retrieved from https://www.who.int/news-room/factsheets/detail/schizophrenia
} 
that need to be investigated and concludes with this study's diverse future scope.

\section{Related Work}

As we know that schizophrenia is a neurodevelopmental disorder in which a patient suffers from different symptoms at different stages. Previous researches have shown that neurotransmitters play a vital role in causing schizophrenia. According to the current progress in technologies, it is proved that a single neuron can produce several neurotransmitters (Brownstein et al., 1974). Thus, it is quite important to study different chemical and enzyme levels in different brain parts. Domino et al. (1973) discussed the regional distribution of enzyme activities in the brain in three groups, deceased mentally healthy people, chronic schizophrenics, and organic brain syndrome patients. The authors studied the regional brain protein concentration, regional brain total cholinesterase activity, regional brain acetylcholinesterase activity, regional brain pseudocholinesterase activity, regional brain choline acetyltransferase activity, regional brain monoamine oxidase activity, and regional brain Nmethyltransferase activity of all the three groups. In comparison, they concluded that there were some differences in the enzyme level in all three groups, although the study was preliminary and needed further validations.

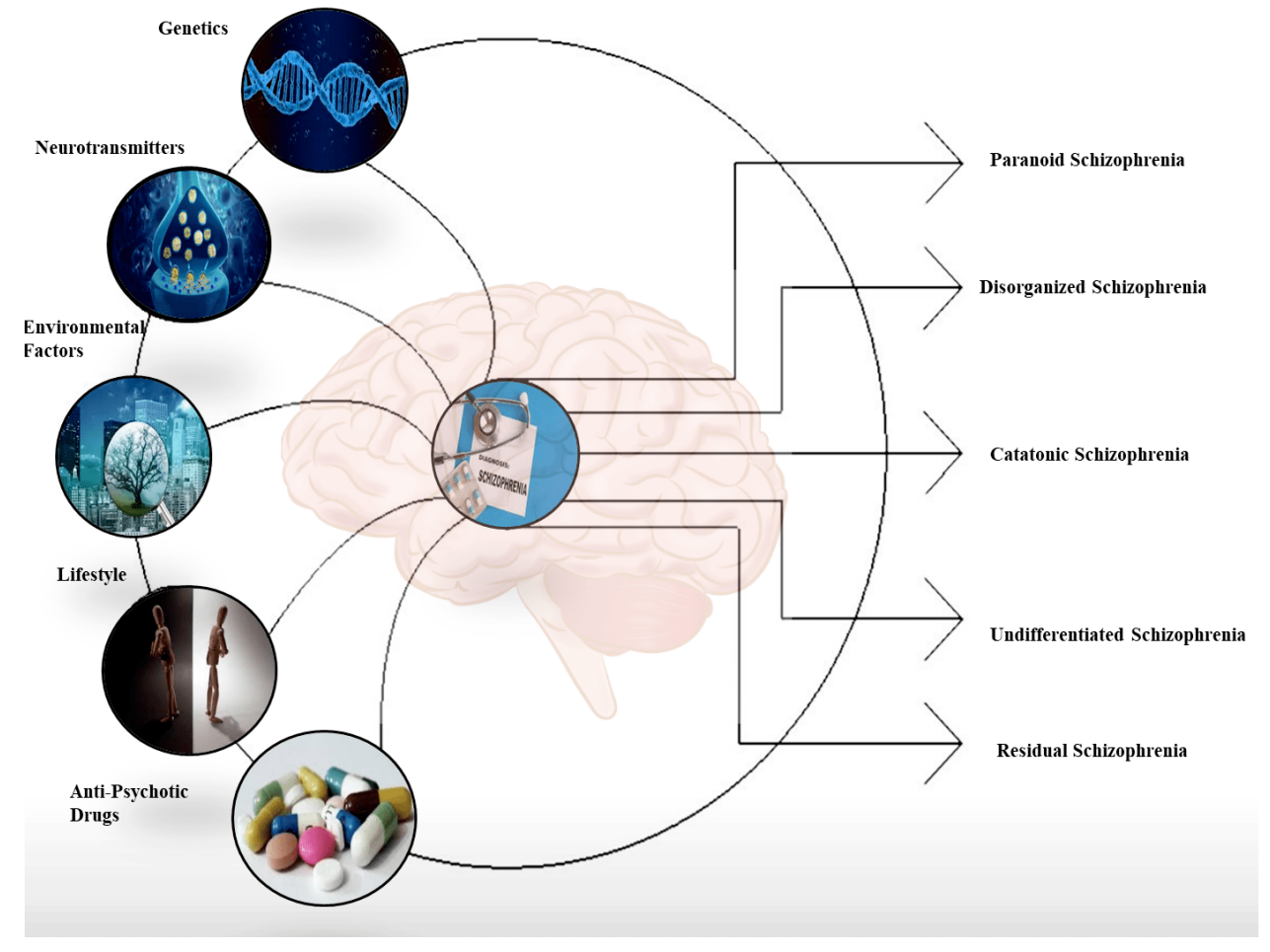

Fig. 1. Various factors which cause schizophrenia: factors like genetics, lifestyle, environmental factors, antipsychotic drugs, and most important neurotransmitters lead to various

types of schizophrenia. 
Table 1. Various chemical groups and their examples.

\begin{tabular}{|ll|}
\hline $\begin{array}{l}\text { Chemical group } \\
\text { CholineEster } \\
\text { Monoamines }\end{array}$ & Example \\
Catechol & Acetylcholine \\
Indole & $\begin{array}{l}\text { Dopamine, neradrenaline (adrenaline) } \\
\text { 5-Hydroxytryptamine (5-HT, Serotonin) }\end{array}$ \\
Imidazole & Histamine (HIST) \\
Amino Acid & Glutamate \\
Acidic Basic & 7-Aminobutyric acid, Glycine \\
Basic & Enkephalins, Endorphins, Cholecystokinin, \\
Peptides & SustanceP \\
& Adenosinetriphosphate, adenosine \\
Purines & Pregnenalone, dehydraepiandrosterone \\
Steroids & (A gas but it is always in solution in the brain) \\
Nitric Acid & Prostaglandins \\
Elcosanoids & \\
\hline
\end{tabular}

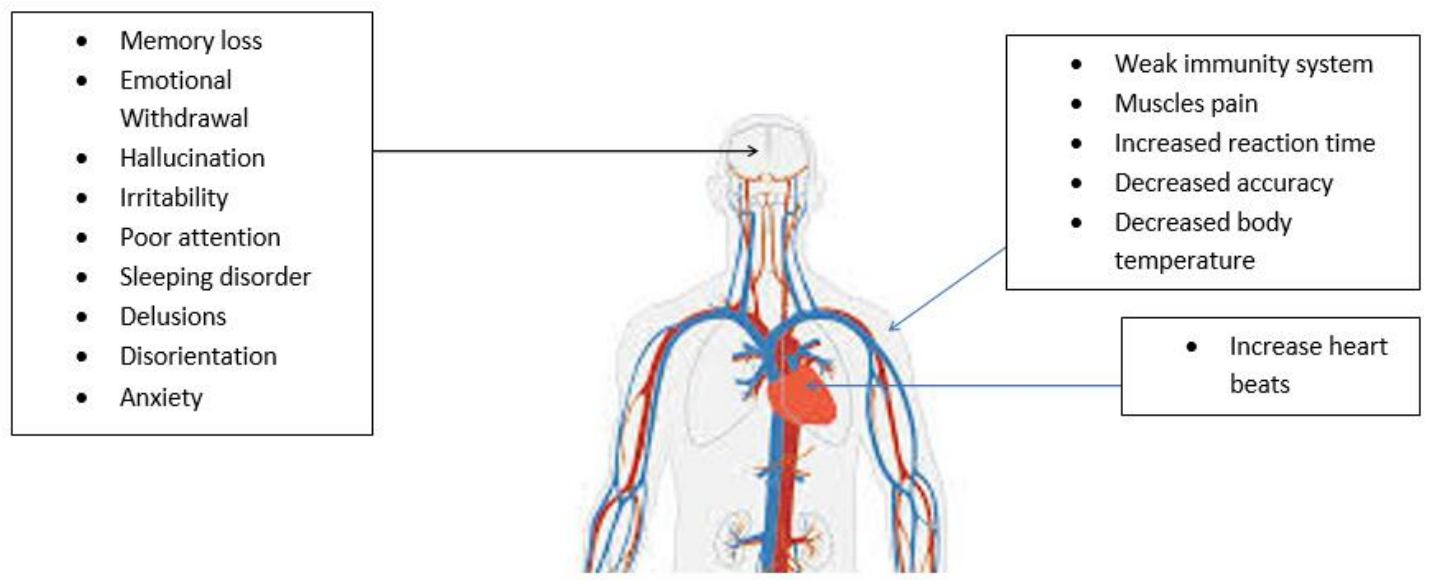

Fig. 2. Various symptoms of schizophrenia: There are various symptoms related to schizophrenia which could be broadly divided into three parts

i) brain-related; ii) body-related and iii) brain-related.

Schizophrenia is mainly about symptoms, and according to the reported literature (Arya et al., 2018; Schultz et al., 2007), these symptoms could be characterized into three types: positive, negative, and acute. Positive symptoms comprise hallucinations, and the negative symptoms include the effect of fathering and loss of every emotional sense, whereas acute symptoms involve loss of memory. (Schultz et al. (2007) also discussed the treatment, including meditation and regular visits to a 
psychologist. However, the patient also has physical changes like weight gain or loss, diabetes, and having suicidal thoughts. They have also mentioned various medications given to patients at different stages of schizophrenia. However, Arya et al. (2018) state that the abnormality of the dopamine replicators is the major reason for the symptoms of schizophrenia in a patient. They also state the different roles played by various parts of the brain in patients who have schizophrenia. Several brain regions are involved in neurotransmitters, such as dopamine, which involves the mesolimbic and mesocortical parts of the brain, and serotonin involves the prefrontal cortex part of the brain. Figure 2 summarizes some major symptoms observed in schizophrenia.

Lieberman \& Koreen. (1993) mentioned the role of neurotransmitters in schizophrenia. The authors have mentioned various neurotransmitters like dopamine, homovanillic acid, plasma homovanillic acid, serotonin, norepinephrine, multivariate analyses, and peptides phospholipids, growth hormone, prolactin, cortisol, and adrenocorticotrophic hormones, and thyroid releasing hormones. However, due to the lack of other parameters, they could not conclude with any final remarks. They stated that the biological substrates are responsible for the pathogenesis of schizophrenia.

Similarly, Howes et al. (2017) show that dopamine plays a significant role in the symptoms of schizophrenia. They mentioned the connection between genes, stress, and dopamine in the development of schizophrenia. They concluded that in schizophrenia, dopamine is released in the blunt cortex. There is increasing evidence that alteration of cortical function is associated with striatal dopamine hyperactivity in individuals with prodromal symptoms. It indicates a central role in corticostriatal deregulation. It is needful to rationally design drugs that target the pathophysiology underlying the disorder's onset to treat it or possibly prevent it.

Factors like genetic, early environment, lifestyle, and various drugs have been listed as the reasons for diabetes in a person. However, Holt et al. (2004) stated that due to metabolic syndrome in schizophrenia, the patients often have diabetes. The authors also mentioned that antipsychotic drugs like Clozapine, Olanzapine, and Risperidone tend to cause weight gain, due to which the patient suffering from diabetes might increase the same. They have referred various patients' reports from the USA, Sweden, Canada, and the UK across all ages and found a link between schizophrenia and diabetes. Finally, they have concluded that there is a need to take special care of patients with schizophrenia and diabetes to avoid their physical health's long-term effects. They also suggested implications of psychiatric care, screening for diabetes, and reconsideration from diabetologists to treat diabetes in patients who have schizophrenia.

Neurotransmitters play an essential role inmaintaining homeostasis for the entire body. Snyder et al. (1974) stated that it was challenging to diagnose schizophrenia in the early '90s. It was necessary to understand which neurotransmitters played a significant role in its characteristics. The authors mentioned the role of effective drugs like Phenothiazines and Catecholamines, stimulants, various psychedelic drugs, and multiple combinations of drugs responsible for 
schizophrenia. Similarly, Mittal et al. (2017) studied the role of numerous neurotransmitters such as norepinephrine, epinephrine, dopamine, and serotonin in the central nervous system pathophysiology. They not only control the blood flow but also affect the neurological system of our body. The authors have reviewed the previous works and concluded using exogenous hormones and psychotropic medications to improve symptomology.

Schizophrenia is mainly about symptoms, and according to the reported literature (Arya et al., 2018; Schultz et al., 2007), these symptoms could be characterized into three types: positive, negative, and acute. Positive symptoms comprise hallucinations, and the negative symptoms include the effect of fathering and loss of every emotional sense, whereas acute symptoms involve loss of memory. Schultz et al. (2007) also discussed the treatment, including meditation and regular visits to a psychologist. However, the patient also has physical changes like weight gain or loss, diabetes, and having suicidal thoughts. They have also mentioned various medications given to patients at different stages of schizophrenia. However, Arya et al. (2018) state that the abnormality of the dopamine replicators is the major reason for the symptoms of schizophrenia in a patient. They also state the different roles played by various parts of the brain in patients who have schizophrenia. Several brain regions are involved in neurotransmitters, such as dopamine, which involves the mesolimbic and mesocortical parts of the brain, and serotonin involves the prefrontal cortex part of the brain. Figure 2 summarizes some major symptoms observed in schizophrenia.

Lieberman \& Koreen. (1993) mentioned about the role of neurotransmitters in schizophrenia. The authors have mentioned various neurotransmitters like dopamine, homovanillic acid, plasma homovanillic acid, serotonin, norepinephrine, multivariate analyses, and peptides phospholipids, growth hormone, prolactin, cortisol, and adrenocorticotrophic hormones, and thyroid releasing hormones. But due to the lack of other parameters, they could not conclude with any final remarks. They stated that the biological substrates are responsible for the pathogenesis of schizophrenia.

Similarly, Howes et al. (2017) show that dopamine plays a significant role in the symptoms of schizophrenia. They mentioned the connection between genes, stress, and dopamine in the development of schizophrenia. They concluded that in schizophrenia, dopamine is released in the blunt cortex. There is increasing evidence that alteration of cortical function is associated with striatal dopamine hyperactivity in individuals with prodromal symptoms. It indicates a central role in corticostriatal deregulation. It is needful to rationally design drugs that target the pathophysiology underlying the disorder's onset to treat it or possibly prevent it.

Factors like genetic, early environment, lifestyle, and various drugs have been listed as the reasons for diabetes in a person, but Holt et al. (2004) stated that due to metabolic syndrome in schizophrenia, the patients often have diabetes. The authors also mentioned that 
antipsychotic drugs like Clozapine, Olanzapine, and Risperidone tend to cause weight gain, due to which the patient suffering from diabetes might increase the same. They have referred various patients' reports from the USA, Sweden, Canada, and the UK across all ages and found a link between schizophrenia and diabetes. Finally, they have concluded that there is a need to take special care of patients with schizophrenia and diabetes to avoid their physical health's long-term effects. They also suggested implications of psychiatric care, screening for diabetes, and reconsideration from diabetologists to treat diabetes in patients who have schizophrenia.

Neurotransmitters play an essential role in maintaining homeostasis for the entire body. Snyder et al. (1974) stated that it was challenging to diagnose schizophrenia in the early '90s. It was necessary to understand which neurotransmitters played a significant role in its characteristics. The authors mentioned the role of effective drugs like Phenothiazines and Catecholamines, Stimulants, various psychedelic drugs, and multiple combinations of drugs responsible for schizophrenia. Similarly, Mittal et al. (2017) studied the role of numerous neurotransmitters such as norepinephrine, epinephrine, dopamine, and serotonin in the central nervous system pathophysiology. They not only control the blood flow but also affect the neurological system of our body. The authors have reviewed the previous works and concluded using exogenous hormones and psychotropic medications to improve symptomology.
Previous shreds of evidence (Gurevich \& Joyce, 1997; Kapur and Remington, 1996; Carlsson \& Carlsson, 1990; Moghaddam, 2003) suggest that other than dopamine, there has been some role of serotonergic and glutamatergic systems that is relevant to schizophrenia and its treatment. Thus, Shah and GonzalezMaeso. (2019) studied the role of serotonergic and glutamatergic in the pathophysiology of schizophrenia. These receptors can modulate each other. The authors stated various pieces of evidence of the crosstalk between serotonergic and glutamatergic receptors. They concluded that the patients are unresponsive to chronic antipsychotic drugs due to the downregulation of serotonergic and glutamatergic neurotransmitters' receptors. Focusing on the role of all the various types of neurotransmitters, Kraal et al. (2019) stated amino acid's role, i.e., glutamate. The authors noted that glutamate is associated with multiple somatic and psychiatric disorders and also with schizophrenia. They concluded that the diet parameters have a strong link. Thus, they suggested the examination of the patient's diet and changed it as prerequirement.

Other than symptoms, there are some significant side effects of schizophrenia, which have the link between insomnia and schizophrenia (Monti \& Monti, 2004; Gupta \& Kulhara, 2010). They have studied various age groups and their sleeping pattern. After considering the previous findings, they concluded that the change in sleeping during schizophrenia is due to the over-activated dopaminergic system. The hypothesis states that the dopamine receptor and neurotransmitter 
are unbalanced in specific brain areas. However, there are various other neurotransmitters like apomorphine, bromocriptine also which affect the sleeping pattern.

\section{Neurochemistry of Schizophrenia Brain}

3.1 Chemicals involved in the functioning of a healthy brain:

The brain controls all the functions of the body. The entire brain has so many chemicals that command every action, reaction, and emotion. Inter-chemical communication properly is vital; otherwise, they cause a chemical imbalance in the brain resulted in different psychological and neurological problems. Table 2 shows various chemicals and emotions which they primarily control.

3.2 Difference between neurotransmitter, brain enzymes, and hormones:

While talking about neurotransmitters, brain enzymes, hormones, and other brain chemicals, we often get confused between their roles and proper definition. Thus, to understand the difference, awareness of the exact meaning of each one of them is necessary. As stated in the oxford dictionary edition 2018 (Dictionary, 2018): Neurotransmitters: "A chemical substance which is released at the end of a nerve fiber by the arrival of a nerve impulse and, by diffusing across the synapse or junction, effects the transfer of the impulse to another nerve fiber, a muscle fiber, or some other structure" (Dictionary, 2018).

Hormones: "A regular substance produced in an organism and transported in tissue fluids such as blood or sap to stimulate specific cells or tissues into action" (Dictionary, 2018).

Enzymes: "A substance produced by a living organism which acts as a catalyst to bring about a specific biochemical reaction" (Dictionary, 2018).

Although, as per the definitions, both neurotransmitters and hormones are responsible for chemical balance in the human brain, they both differ in some way or another. As hormones get released from their respective glands into the bloodstream and directly go to their distant targets, neurotransmitters are released as a signal from tip to tip of the nerve cell (Nicholls et al., 2001).

\subsection{Neurotransmitters or enzymes involved in schizophrenia}

There are various neurotransmitters involved in schizophrenia. Table 3 summarizes various literature and the neurotransmitters they mentioned with schizophrenia.

The major neurotransmitters are as follows.

- Dopamine (Berridge \& Robinson, 1998; Abboud et al., 2017; Bowers, 1974)

- Serotonin (Owens \& Nemeroff, 1994)

- GABA (Lydiard, 2003; Abboud et al., 2017)

- Glutamate (Choi and Rothman, 1990) 
The disorder of neuropeptides like neurotensin \& cholecystokinin (Ressler \& Nemeroff, 1999) also plays an important role in the same (Werner \& Covenas, 2013).

3.4 The effect of neurotransmitters in different parts of the brain in schizophrenia

We know that neurotransmitters play such a vital role in transmitting nerve signals and controlling various human emotions. It is essential to identify the source of the neurotransmitters source and their effects in different brain parts, which trigger different symptoms in schizophrenia. Every neurotransmitter trigger different schizophrenia symptoms. Figure 3 shows various neurotransmitters in various parts of the brain.

(i) Dopamine (Seeman, 1980; Wise, 2004)

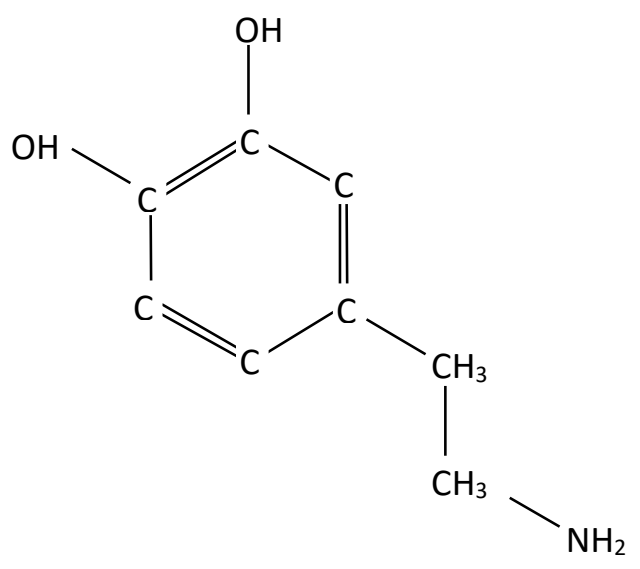

Dopamine is the most vital neurotransmitter of the human brain. It is a signal transmitter of the nerves. It belongs to the family of catecholamine and phenethylamine families, having the chemical formula of $\mathrm{C}_{8} \mathrm{H}_{11} \mathrm{NO}_{2}$. It is an organic chemical that can act both as a hormone and neurotransmitter in the human brain.

Any interference in the dopamine path can lead to various disorders, and one of the most common is memory loss. The difficulties in retaining memory to perform everyday tasks are observed in schizophrenia. During schizophrenia, the patient cannot perform typical day-to-day tasks as the signals are not transmitted adequately (Seeman, 1980). Physiopathologically, it is believed that increased dopaminergic (or dysregulated) transmission in the mesolimbic pathway from the ventral tegmental region to the nucleus accumbens plays a crucial role in the generation of positive symptoms (Feifel et al., 2016). Figure 4 shows the difference between the standard and increased dopaminergic transmission in a healthy human and a patient who has schizophrenia.

Dopamine is someway responsible for the regulation of movement, attention, learning, and emotional responses. Schizophrenia affects how a person thinks, feels, and acts (Gupta \& Kulhara, 2010), making dopamine more responsible for the disorder. Monti \& Monti. (2004) stated the effect of schizophrenia on the patient's sleeping pattern from early-stage to the final stage. The authors discussed the overactivity of the dopaminergic system in schizophrenia, due to which a person suffers a different sleeping pattern. Literature (Laruelle et al., 1999) shows that a hyperdopaminergic state is present in schizophrenia during the initial episode 
and relapses, but not in a period of remission. Again, the diminished dopaminergic neurotransmissions in the human brain are responsible for major depression (Dunlop \& Nemeroff, 2007).
Flor-Henry. (1969) In his paper about the Flor-Henry proposal on schizophrenia as a dysfunction of the left temporal lobe, connect the neurochemicals with the FlorHenry proposal. The author has done

Table 2. Role of Various Neurotransmitters in Brain

\begin{tabular}{lll}
\hline Chemicals & Role & Reference(s) \\
Dopamine & Motivation and movement but also handles memory and focus & Berridge and Robinson. (1998) \\
Oxytocin & Feeling of bonding & IsHak et al. (2011) \\
Serotonin & Feeling of hunger & Owens and Nemeroff. (1994) \\
Endorphins & Feeling of pain and discomfort & Amir et al. (1980) \\
Glutamate & Learning and memory & Choi and Rothman. (1990) \\
GABA & Slowdowns the nervous activities & Lydiard. (2003) \\
Acetylcholine & Learning and memory & Hasselmo. (2006) \\
Cannabinoids & Regulating nausea and vomiting & Malik et al. (2015) \\
Opioids & Modify the other central nervous system & Froehlich. (1997) \\
Epinephrine & Provide extra energy to body & Axelrod and Tomchick. (1958) \\
Norepinephri & Helps in narrowing the blood vessels so that the blood pressure increases & Ressler and Nemeroff. (1999) \\
\hline
\end{tabular}

Table 3. List of important studies mentioning various neurotransmitters: studies from late '90s to early '20s all studies have broadly mentioned the role of dopamine, GABA, and glutamate and very few about serotonin in the study of schizophrenia.

\begin{tabular}{lll}
\hline Paper & Year & Chemical(s)Mentioned \\
1.Bowers. (1974) & 1974 & Dopamine \\
2.Carlsson. (1978) & 1978 & Dopamine \\
3.Meltzer et al. (1981) & 1981 & Dopamine \\
4.Korpi et al. (1986) & 1986 & Serotonin \\
5.Toru et al. (1988) & 1988 & Dopamine and Glutamate \\
6.Choi and Rothman. (1990) & 1990 & Glutamate \\
7.Lieberman and Koreen. (1993) & 1993 & Dopamine, Serotonin, Neuropipehrine, Glutamate \\
8.Owens and Nemeroff. (1994) & 1994 & Serotonin \\
9.Kapur and Remington. (1996) & 1996 & Serotonin and Dopamine \\
10.Carlsson et al. (2001) & 2001 & Dopamine, GABA, Glutamate \\
11.Watanabe et al. (2002) & 2002 & GABA \\
12.Moghaddam. (2003) & 2003 & Glutamate \\
13.Menschikov et al. (2016) & 2016 & GABA, Glutamate \\
14.Abboud et al. (2017) & 2017 & Dopamine and GABA \\
15.McCutcheon et al. (2019) & 2019 & Dopamine \\
16.Swanton. (2020) & 2020 & Dopamine, GABA, Glutamate \\
17.Peitl et al. (2020) & 2020 & Serotonin \\
18.McCutcheon et al. (2020) & 2020 & Dopamine, GABA, Glutamate \\
\hline
\end{tabular}


several tests on the brain tissue of people who have schizophrenia before death. The author also concluded a considerable increase of dopamine in the left cerebral hemisphere of the people who have schizophrenia (Reynolds, 1983).

Laruelle et al. (2003) discussed the interaction between glutamate and dopamine. The authors reviewed the literature and discussed dopamine and glutamate's role during schizophrenia, including loss of senses, motion, memory, and depression. A low level of dopamine is linked with various brain disorders like schizophrenia (Birtwistle \& Baldwin, 1998), Parkinson's disease (Birtwistle \& Baldwin, 1998), depression (Brown \& Gershon, 1993) and psychosis (Tost et al., 2010).

(ii) Oxytocin (MacDonald and Feifel, 2012; Averbeck et al., 2012)

Oxytocin $\left(\mathrm{C}_{43} \mathrm{H}_{66} \mathrm{~N}_{12} \mathrm{O}_{12} \mathrm{~S}_{2}\right)$ is one of the most vital peptide hormones in the human brain as it helps regulate social bonding, sexual desire, childbirth, and menstruation. This hormone is released from the posterior pituitary and is produced in the hypothalamus gland. Oxytocin has long been studied as being able to play a role in the pathophysiology of schizophrenia, given its effects on cognition, memory, and social functioning (Cochran et al., 2013).

During schizophrenia, when the patient loses their social bonding and sexual desires, different studies show that it is due to interference in the oxytocin path (Bartz et al., 2011). Keri et al. (2009) studied schizophrenia patients and normal healthy subjects. They stated that schizophrenia patients have a low level of oxytocin due to which negative symptoms develop, such as decreased trust, social withdrawal, isolation, and flattened affect. However, on the other hand, the authors also concluded that no positive symptoms were developed because of the low level of oxytocin. Averbeck et al. (2012) stated that the beneficial effects of oxytocin on the social behavioral tasks of the schizophrenia patient. The authors studied approximately thirty patients who have schizophrenia before and after the administration of oxytocin. They concluded that after the administration of oxytocin, the patients were able to recognize emotions. This hormone is also responsible for treating schizophrenia because of its antipsychotic potential (MacDonald \& Feifel, 2012).

Feifel et al. (2016) stated the oxytocin effects causing positive, negative, and cognitive symptoms in schizophrenia. The authors found that oxytocin does not cause any such positive symptoms in schizophrenia, but symptoms such as partner preference in females and lack of social interaction. These negative symptoms are due to change in oxytocin levels. Oxytocin also causes cognitive defects, such as social recognition and social perception, during schizophrenia.

(iii) GABA (Wassef et al. 2003)

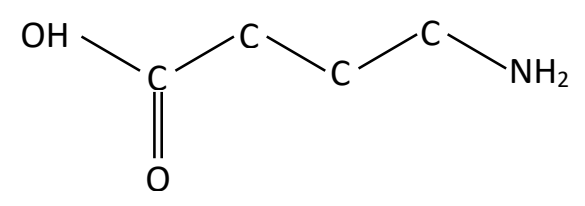

GABA (Gamma-Aminobutyric Acid$\mathrm{C}_{4} \mathrm{H}_{9} \mathrm{NO}_{2}$ ) is a gamma-amino acid that is 
butanoic acid $\left(\mathrm{C}_{4} \mathrm{H}_{8} \mathrm{O}_{2}\right)$ with the amino substitution. It is the most widely spread neurotransmitter in the central nervous system and the cortex region. It controls all the cortical functions. This neurotransmitter also controls anxiety and its feeling, as GABA attaches to a protein in our brain, known as a GABA receptor. It produces a calming effect in the mind.

Low level of GABA has various effects on the body. During some medical conditions, the GABA level is reduced, causing multiple difficulties for the human body. Petroff et al. (1996) stated that a low GABA level is associated with poor seizure control. Carlsson et al. (2001) studied other monoamines and glutamate and GABA so that a new direction is open for a better understanding of schizophrenia. At a very initial stage, it was believed that no neurotransmitter is responsible for the symptoms of schizophrenia other than dopamine. Still, glutamate and GABA could deal with more general defects.

The link between the sleep disorder and schizophrenia is mentioned in the literature (Kaskie et al., 2017) that involved risks to patients with sleeping disorders. Monti and Monti. (2005) suggested that during schizophrenia, insomnia occurs partly due to the dopaminergic system's overactivity, but there are chances that the GABAergic system could also be involved in this disorder.

As stated earlier, the primary neurotransmitter in schizophrenia is dopamine. Thus, studying the relation between GABA and dopamine in schizophrenia is an important area. After considering various previous data and literature, Garbutt \& van Kammen. (1983) stated that it was previously believed that GABA might be useful in treating schizophrenia, as it inhibits dopaminergic activity. However, the recent data showed a new direction to the authors, which revealed that GABA has the opposite effect on the dopaminergic system.

Due to the lack of several parameters and technology, this study could not propose relevant results. Other studies (Fukumaki \& Shibata, 2003; Abboud et al., 2017) mentioned the role of GABA in schizophrenia. However, the result of the interaction between GABA and other neurotransmitters is yet to be disclosed.

(iv) Glutamate (Meyer, 2014)<smiles>NC(CCC(=O)O)C(=O)O</smiles>

Glutamate (C5H8NO4) is the principal' excitatory' neurotransmitter in the brain, which helps activate neurons and other brain cells. About $60 \%$ of neurons contain glutamate, and almost all have a type of glutamate receptor (Zhou \& Danbolt, 2014). Glutamate also helps in the synthesis of GABA, which is again one of the most vital neurotransmitters in the central nervous system (Petroff, 2002; Watanabe et al., 2002). Glutamate contributes to the development of the 
prenatal brain and the child. As the brain matures, the crucial roles are learning and memory. Glutamate is essential for longterm potentiation (LTP), a process by which new information or skills are stored for later use (Meyer, 2014).

During schizophrenia, the patient cannot retain memory and has difficulties forming skills that he or she already knew. It directly shows that there is something linked with the role of glutamate. Different studies (Fukumaki \& Shibata, 2003; Meyer, 2014) found that people at risk of developing schizophrenia may have too much glutamate activity in some regions of the brain. As the disease progresses, these areas of the brain may not have enough glutamate activity.

Laruelle et al. (2003) have mentioned the relation between glutamate, dopamine, and schizophrenia. The authors studied various literature and had different tests on animals. They concluded by stating that abnormalities in schizophrenia are due to strong interconnection between glutamate and dopamine transmissions.

Stone et al. (2007) studied the relationship between dopamine and glutamate deregulation in schizophrenia and its connection. The authors stated that if glutamate is responsible for schizophrenia, its release is modulated by the dopamine system or leads to downstream effects in the dopamine system. Thus, the symptoms of schizophrenia or any psychotic symptoms are noticed. The authors also said that there are high chances that dopamine and glutamate disorder give rise to different symptoms, such as glutamate could be responsible for negative symptoms, and dopamine is responsible for positive traits.
They concluded that further studies are needed for some specific outcomes.

Various studies (Fukumaki \& Shibata, 2003; Meyer, 2014) have stated that glutamate is responsible for negative symptoms in schizophrenia. Tsai \& Coyle. (2002) mentioned glutamatergic mechanisms in schizophrenia and noted that post-mortem studies show that glutamate receptors were altered in schizophrenia. They concluded that the hypofunction glutamate receptors might participate in schizophrenia.

Various studies (Javitt, 2010; Abboud et al., 2017) still in progress where the relation between glutamate, dopamine, and GABA are being studied for a better outcome.

(v) Serotonin

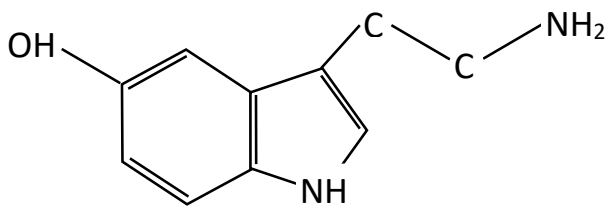

Serotonin $\left(\mathrm{C}_{10} \mathrm{H}_{12} \mathrm{~N}_{2} \mathrm{O}\right)$ is a chemical of nerve cells. It sends signals between the nerve cells. Serotonin is found mainly in the digestive system, although it is also found in blood platelets throughout the central nervous system. It is a monoamine neurotransmitter. This neurotransmitter is responsible for the feeling of well-being and happiness (Sternbach, 1991; Jacobs \& Azmitia, 1992). Sternbach. (1991) stated that serotonin syndrome is most commonly the result of the interaction between serotonergic agents and monoamine oxidase inhibitors. This 
syndrome's significant features are changes in mental status, restlessness, diaphoresis, shivering, and tremor.

Bleich et al. (1988) stated that the serotonin's findings show that it is responsible for the negative symptoms like the degradation of the brain and chronicity. Similarly, Igbal \& van Praag. (1995) observed a link between serotonergic defects and schizophrenia. The authors concluded with three points, firstly, the disorder in the serotonin system causes brain disorders; secondly, the tentative shreds of evidence have been found to state the link between serotonin and schizophrenia; and finally, the natural occurrence of schizophrenia due to serotonin is yet to explore.

When stating about different neurotransmitters, there are high chances that they interact with dopamine, due to which various symptoms are observed in schizophrenia. Kapur \& Remington. (1996) mentioned in their paper about serotonin-dopamine interaction and its relevance to schizophrenia. The authors surveyed more than 100 articles and found that the serotonin system inhibits dopaminergic function at the origin level, although this is only noticed in a narrow range. They concluded that serotonergic modulation of dopaminergic function provides a viable solution mechanism to improve therapies in schizophrenia, but many remain unclear, and further studies are required. Ohuoha et al. (1993) also stated that serotonin's role in schizophrenia is still not clear, and the interaction between serotonin and dopamine needs more in-depth study and tests.

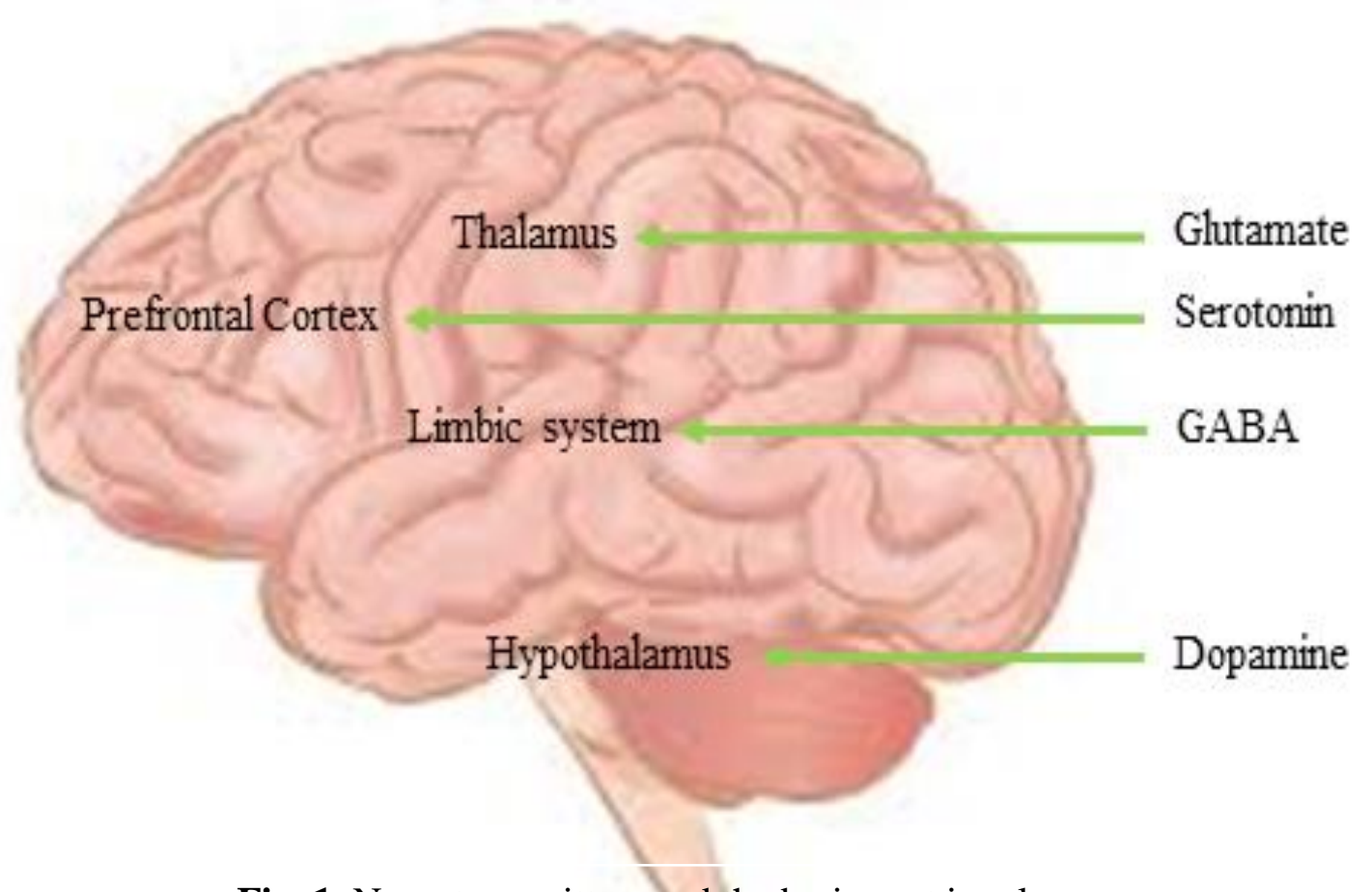

Fig. 1. Neurotransmitters and the brain part involve 


\section{Discussion}

As discussed, schizophrenia (Bateson et al., 1956; Chatterjee et al., 2018a, 2020a) is caused due to various reasons like genetics, environment, a chemical imbalance in the brain, and structural deformities. Due to this disorder, many factors have been reported, from psychotherapy to autoimmune to genetic factors. The biggest question was to investigate the role and effect of neurotransmitters in schizophrenia and find all the responsible neurotransmitters. This paper reviews the literature rigorously, discusses state-of-the-art research and mentions critical neurotransmitters responsible for various symptoms in schizophrenia. It has widely known that the symptoms of schizophrenia are divided majorly into two types, i.e., positive and negative symptoms (Schultz et al., 2007). The words like positive and negative could be misleading with the good and bad symptoms. In schizophrenia, the positive symptoms refer to the characteristics added to someone's state of being, and the negative ones refer to the characteristics that are removed from someone's state of being.

After identifying the critical neurotransmitters involved in schizophrenia, the difference between neurotransmitters, hormones, enzymes, and brain chemicals needs to be discussed. Here, this paper describes the difference in the functionality of brain chemicals, hormones, neurotransmitters, and enzymes. Often, the neurotransmitters and hormones are considered the same as they are both responsible for the brain's chemical balance. However, they both are different and cannot be used interchangeably. Our primary focus was to identify the role of neurotransmitters in schizophrenia. Thus, after a brief comparison between neurotransmitters and hormones, the paper holds a detailed explanation of different neurotransmitters responsible for the disorder and presents their interconnection. While mentioning different chemicals in the human brain, we have also noted the important role of each chemical in the healthy human brain to understand the changes a brain might undergo if any chemical pathway is affected (Nicholls et al., 2001).

However, the primary objectives were to identify neurotransmitters' role and their effects in different parts of the brain during schizophrenia. After the critical literature review, it may be concluded that among dopamine (Seeman, 1980), serotonin (Bleich et al., 1988), glutamate (Meyer, 2014), GABA (Wassef et al., 2003), and oxytocin (MacDonald \& Feifel, 2012), dopamine is the most vital neurotransmitter in the schizophrenia. Dopamine gets the maximum attention, followed by glutamate, serotonin, GABA, and oxytocin. We have identified several insights into the interconnection between these neurotransmitters after critically reviewing the most relevant literature. Oxytocin also holds properties that could help treat schizophrenia (MacDonald \& Feifel, 2012). Belonging to the family of catecholamine and phenethylamine, dopamine is an organic compound that 
can act both as hormone and neurotransmitter in the human brain. This compound is responsible for various human activities (Berridge \& Robinson, 1998) like motivation, movement, memory, and focus. If this compound's path is affected in the human brain, all the mentioned activities get disturbed, especially an individual's memory. Change in dopamine is not just linked with schizophrenia but also with various disorders like Parkinson's disease (Birtwistle \& Baldwin, 1998), depression (Brown \& Gershon, 1993) and psychosis (Tost et al., 2010). During the literature survey, we came across the fact that in schizophrenia, there is some interaction between glutamate and dopamine, but an in-depth study is still required.

The neurotransmitter that activates all neurons is glutamate (Zhou \& Danbolt, 2014). Glutamate is mainly responsible for learning and memory, which helps store new skills and information. Though dopamine is responsible for the loss of memory in schizophrenia, and it was found that there is a direct link with glutamate when the patient is not able to perform the everyday activity and has trouble in restoring old or learned skills and information (Meyer, 2014). It was noticed that there is a relationship between glutamate and dopamine in schizophrenia, in which the dopamine system modulates glutamate. Scientists have talked about the different symptoms, which could be due to the interconnection between glutamate and dopamine, as glutamate causes negative symptoms and dopamine causes positive symptoms. On the other hand, glutamate and GABA interaction are less. However, glutamate helps in the synthesis of GABA, which is again one of the vital neurotransmitters in the central nervous system and an essential neurotransmitter in schizophrenia (Javitt, 2010; Abboud et al., 2017).

The most widely spread neurotransmitter in the human central nervous system and cortex region is glutamate. It controls most of the functions and maintains the central nervous system. This neurotransmitter also controls anxiety and feeling of the same. It produces a calming effect on humans.

The links between GABA and dopamine, and GABA and glutamate, have many areas yet to be known (Fukumaki \& Shibata, 2003; Abboud et al., 2017). GABA is also responsible for the sleeping disorder in patients noticed during schizophrenia, but this needs more research and data for better confirmation.

The feeling of well-being, hunger and happiness is controlled by serotonin (Owens \& Nemeroff, 1994). This neurotransmitter is found at various places in the body, mainly in the digestive system, because it controls the feeling of hunger. After numerous investigations, it was found that serotonin is responsible for the negative symptoms during schizophrenia (Bleich et al., 1988). The link between serotonin and dopamine is yet to be explored in detail because after comparing the individual effects, it is noted that they might interact during schizophrenia (Kapur \& Remington, 1996).

To date, very few studies have been conducted on the role of oxytocin in schizophrenia (Cochran et al., 2013). As 
oxytocin is responsible for bonding in humans, its low level in the human brain may cause social withdrawal. There are not many studies related to it. Oxytocin is also used in treating psychotic disorders due to its antipsychotic potential. We believe this could be a potential research area where further in-depth research could draw some light.

From the early researches on schizophrenia, dopamine is considered the most crucial neurotransmitter in schizophrenia. With new details of various neurotransmitters involved in schizophrenia, it is essential to know how they link to each other and their several effects.

We found that the available literature on the interconnections between different neurotransmitters in schizophrenia is still significantly less. The neurotransmitters other than dopamine may also be the reasons behind various symptoms that prevail in schizophrenia. As we find that dopamine is responsible for many more brain disorders, linking the other neurotransmitters with dopamine will help us better understand.

While studying various symptoms and neurotransmitters, we noticed that some other health problems are also linked with changes in neurotransmitters in schizophrenia. We believe this could be a new direction to diagnose schizophrenia. We think these research areas could lead us to a new course in the field of schizophrenia. Firstly, linking various neurotransmitters with each other, especially those responsible for the body's same activity. Secondly, to explore all the problems other than psychotic disorders when different neurotransmitters are affected. Thirdly, the role of various neurotransmitters involved at varying levels of schizophrenia.

It is quite clear from this study that schizophrenia is caused due to the chemical imbalance in the brain. If not treated properly, it could be dangerous, as the mortality rate primarily indicates suicide cases due to rises of symptoms to an unbearable extent.

We have identified many potential research gaps in this domain that deal with neurotransmitter studies in schizophrenia. For further studies, it is recommended that one may investigate the gaps in the studies of interconnection between various neurotransmitters in schizophrenia and find correlations with other disorders. It is also suggested that the researchers may concentrate more on other neurotransmitters in the brain to find any interference between one another. We believe that the linkage between genetically transferred symptoms of schizophrenia may also be studied. Recently, efforts are being made to automate biological studies using computational analysis (Jain and Kumar, 2018). Machine learning (Domingos, 2012; Kumar \& Chatterjee, 2016; Gautam \& Chatterjee, 2020) takes over classical statistical approach in biomedical studies (Colak et al., 2016; Firat et al., 2016; Moses et al., 2015), even in hormonal studies (Rajkumar \& Palanichamy, 2015). Machine learning studies on neuroscience, especially schizophrenia, are quite popular these days (Chatterjee et al., 2018a, 2019). We believe that the incorporation of machine intelligence in the field of enzymatic study of schizophrenia may open up many unknown characteristics of 
metabolites present in the brain of schizophrenia patients.

\section{Conclusion}

As the human brain is the most complex organ, research on the neurochemistry of psychological disorders like schizophrenia was always an exciting area to explore. This paper has explored all the neurotransmitters, enzymes, hormones, and brain chemicals involved in schizophrenia. This paper critically reviews most of the relevant literature in this domain available to date. After the broad literature survey, we have studied and presented a detailed discussion on the role of various neurotransmitters in schizophrenia. This paper identifies that neurotransmitters like dopamine, glutamate, GABA, serotonin, and oxytocin are majorly responsible for schizophrenia, among which dopamine plays the most vital role. Here, we have also investigated the role of different neurotransmitters at various stages of the disorder concerning a patient's symptoms to identify the interconnections between various neurotransmitters. This study successfully identified several unexplored research gaps, potentially essential to understand the neurochemistry of schizophrenia. The unsolved questions mentioned in this paper may pose as a cue for the scope of further studies. This paper throws light on the present understanding of the neurochemistry of the disorder, especially the neurotransmitters.

\section{ACKNOWLEDGMENTS}

The authors acknowledge the Tongmyong University, Busan, South Korea, and JK Lakshmipat University, Jaipur, India, for providing the facility to pursue this study.

\section{References}

Abboud, R.; Noronha, C. \& Diwadkar, V. A. (2017), 'Motor system dysfunction in the schizophrenia diathesis: neural systems to neurotransmitters', European Psychiatry 44, 125-133.

Amir, S.; Brown, Z. W. \& Amit, Z. (1980), 'The role of endorphins in stress: Evidence and speculations', Neuroscience \&Biobehavioral Reviews 4(1), 77-86.

Arya, A.; Sindhwani, G. \& Kadian, R. (2018), 'Neurotransmitter and brain parts involved in schizophrenia', Asian J Pharm Clin Res 11(6), 4-11.

Averbeck, B.; Bobin, T.; Evans, S. \& Shergill, S. (2012), 'Emotion recognition and oxytocin in patients with schizophrenia', Psychological medicine 42(2), 259-266.

Axelrod, J. \& Tomchick, R. (1958), 'Enzymatic o-methylation of epinephrine and other catechols', J Biol Chem 233(3), 702-705.

Bartz, J. A.; Zaki, J.; Bolger, N. \& Ochsner, K. N. (2011), 'Social effects of oxytocin in humans: context and person matter', Trends in cognitive sciences 15(7), 301-309. 
Bateson, G.; Jackson, D. D.; Haley, J. \& Weakland, J. (1956), 'Toward a theory of schizophrenia', Behavioral science 1(4), 251-264.

Beckmann, H.; Franzek, E. \& Sto“ ber, G. (1996), 'Genetic heterogeneity in catatonic schizophrenia: a family study', American journal of medical genetics 67(3), 289-300.

Berridge, K. C. \& Robinson, T. E. (1998), 'What is the role of dopamine in reward: hedonic impact, reward learning, or incentive salience?', Brain research reviews 28(3), 309-369.

Birtwistle, J. \& Baldwin, D. (1998), 'Role of dopamine in schizophrenia and Parkinson's disease', British journal of nursing 7(14), 832-841.

Bleich, A.; Brown, S.-L.; Kahn, R. \& van Praag, H. M. (1988), 'The role of serotonin in schizophrenia', Schizophrenia bulletin 14(2), 297-315.

Bowers, M. B. (1974), 'Central dopamine turnover in schizophrenic syndromes', Archives of General Psychiatry 31(1), 5054.

Brown, A. \& Gershon, S. (1993), 'Dopamine and depression', Journal of Neural Transmission/General Section JNT 91(2-3), 75-109.

Brownstein, M. J.; Saavedra, J. M.; Axelrod, J.; Zeman, G. H. \& Carpenter, D. O. (1974), 'Coexistence of several putative neurotransmitters in single identified neurons of aplysia',
Proceedings of the National Academy of Sciences 71(12), 4662-4665.

Brüne, M. (2003), 'Theory of mind and the role of iq in chronic disorganized schizophrenia', Schizophrenia research 60(1), 57-64.

Carlsson, A. (1978), 'Antipsychotic drugs, neurotransmitters, and schizophrenia', American Journal of Psychiatry 135(2), 164-173.

Carlsson, A.; Waters, N.; Holm-Waters, S.; Tedroff, J.; Nilsson, M. and Carlsson, M. L. (2001), 'Interactions between monoamines, glutamate, and Gaba in schizophrenia: new evidence', Annual review of pharmacology and toxicology 41(1), 237-260.

Carlsson, M. \& Carlsson, A. (1990), 'Interactions between glutamatergic and monoaminergic systems within the basal ganglia-implications for schizophrenia and Parkinson's disease', Trends in neurosciences 13(7), 272-276.

Catts, V. S.; Fung, S. J.; Long, L. E.; Joshi, D.; Vercammen, A.; Allen, K. M.; Fillman, S. G.; Moore, L.; Rothmond, D.; Sinclair, D. et al. (2013), 'Rethinking schizophrenia in the context of normal neurodevelopment', Frontiers in cellular neuroscience 7, 60 .

Chatterjee, I.; Agarwal, M.; Rana, B.; Lakhyani, N. \& Kumar, N. (2018a), 'Biobjective approach for computer-aided diagnosis of schizophrenia patients using fmri data', Multimedia Tools and Applications 77(20), 26991-27015. DOI: 
https://dx.doi.org/10.1007/s11042-018-

$\underline{5901-0}$

Chatterjee, I. (2018b), 'Mean deviation based identification of activated voxels from timeseries fmri data of schizophrenia patients', F1000Research 7(1615). DOI: https://dx.doi.org/10.12688/f1000research. $\underline{16405.2}$

Chatterjee, I.; Kumar, V.; Sharma, S.; Dhingra, D.; Rana, B.; Agarwal, M. and Kumar, N. (2019), 'Identification of brain regions associated with working memory deficit in schizophrenia', F1000Research 8(124).

DOI: https://doi.org/10.12688/f1000research.17 $\underline{731.1}$

Chatterjee, I.; Kumar, V.; Rana, B.; Agarwal, M. \& Kumar, N. (2020a), 'Identification of changes in grey matter volume using an evolutionary approach: an mri study of schizophrenia', Multimedia Systems 26, 383-396. DOI: https://dx.doi.org/10.1007/s00530-020$\underline{00649-6}$

Chatterjee, I.; Kumar, V.; Rana, B.; Agarwal, M. \& Kumar, N. (2020b), 'Impact of ageing on the brain regions of the schizophrenia patients: an fmri study using evolutionary approach', Multimedia Tools and Applications 79(33), 24757-24779. DOI: https://dx.doi.org/10.1007/s11042-020$\underline{09183-\mathrm{z}}$

Chatterjee, I. \& Mittal, K. (2020), 'A concise study of schizophrenia and resting-state fmri data analysis', Qeios
414(599711.2). DOI:

https://dx.doi.org/10.32388/599711.2

Choi, D. W. \& Rothman, S. M. (1990), 'The role of glutamate neurotoxicity in hypoxic-ischemic neuronal death', Annual review of neuroscience 13(1), 171-182.

Cochran, D.; Fallon, D.; Hill, M. and Frazier, J. A. (2013), 'The role of oxytocin in psychiatric disorders: a review of biological and therapeutic research findings', Harvard review of psychiatry 21(5), 219.

Colak, C.; Colak, M. C.; Ermis, N.; Erdil, N. \& Ozdemir, R. (2016), 'Prediction of cholesterol level in patients with myocardial infarction based on medical data mining methods', Kuwait Journal of Science 43(3).

Dictionary, O. E. (1989), 'Oxford English dictionary', Simpson, JA \& Weiner, ESC .

Dictionary, O. E. (2018), 'art n. 1', OED Online. Oxford University Press: Oxford, UK .

Domingos, P. (2012), 'A few useful things to know about machine learning', Communications of the ACM 55(10), 7887.

Domino, E. F.; Krause, R. R. \& Bowers, J. (1973), 'Various enzymes involved with putative neurotransmitters: Regional distribution in the brain of deceased mentally normal, chronic schizophrenics or organic brain syndrome patients', Archives of General Psychiatry 29(2), 195-201. 
Dunlop, B. W. \& Nemeroff, C. B. (2007), 'The role of dopamine in the pathophysiology of depression', Archives of general psychiatry 64(3), 327-337.

Feifel, D.; Shilling, P. D. \& MacDonald, K. (2016), 'A review of oxytocin's effects on the positive, negative, and cognitive domains of schizophrenia', Biological psychiatry 79(3), 222-233.

Firat, F.; Arslan, A. K.; Colak, C. \& Harputluoglu, H. (2016), 'Estimation of risk factors associated with colorectal cancer: an application of knowledge discovery in databases', Kuwait Journal of Science 43(2).

Flor-Henry, P. (1969), 'Psychosis and temporal lobe epilepsy; a controlled investigation', Epilepsia 10(3), 363-395.

Froehlich, J. C. (1997), 'Opioid peptides', Alcohol health and research world 21, 132-135.

Fukumaki, Y. \& Shibata, H. (2003), 'Glutamate receptor genes as candidates for schizophrenia susceptibility', Drug development research 60(2), 137-151.

Garbutt, J. C. \& van Kammen, D. P. (1983), 'The interaction between gaba and dopamine: implications for schizophrenia', Schizophrenia bulletin 9(3), 336-353.

Gautam, A. \& Chatterjee, I. (2020), 'Big data and cloud computing: A critical review', International Journal of Operations Research and Information
Systems (IJORIS) 11(3), 19-38. DOI: https://dx.doi.org/10.4018/IJORIS.202007 $\underline{0102}$

Geisler, D.; Walton, E.; Naylor, M.; Roessner, V.; Lim, K. O.; Schulz, S. C.; Gollub, R. L.; Calhoun, V. D.; Sponheim, S. R. and Ehrlich, S. (2015), 'Brain structure and function correlates of cognitive subtypes in schizophrenia', Psychiatry Research: Neuroimaging 234(1), 74-83.

Gupta, S. and Kulhara, P. (2010), 'What is schizophrenia: A neurodevelopmental or neurodegenerative disorder or a combination of both? a critical analysis', Indian journal of psychiatry 52(1), 21.

Gurevich, E. V. \& Joyce, J. N. (1997), 'Alterations in the cortical serotonergic system in schizophrenia: a post-mortem study', Biological psychiatry 42(7), 529545.

Hasselmo, M. E. (2006), 'The role of acetylcholine in learning and memory', Current opinion in neurobiology $16(6)$, 710-715.

Holt, R.; Peveler, R. \& Byrne, C. (2004), 'Schizophrenia, the metabolic syndrome and diabetes', Diabetic Medicine 21(6), 515-523.

Howes, O. D.; McCutcheon, R.; Owen, M. J. \& Murray, R. M. (2017), 'The role of genes, stress, and dopamine in the development of schizophrenia', Biological psychiatry 81(1), 9-20. 
Igbal, N. \& van Praag, H. M. (1995), 'The role of serotonin in schizophrenia', European Neuropsychopharmacology 5, 11-23.

Insel, T. R. (2010), 'Rethinking schizophrenia', Nature 468(7321), 187.

IsHak, W. W.; Kahloon, M. and Fakhry, H. (2011), 'Oxytocin role in enhancing well-being: a literature review', Journal of affective disorders 130(1-2), 19.

Jacobs, B. L. \& Azmitia, E. C. (1992), 'Structure and function of the brain serotonin system', Physiological reviews 72(1), 165-229.

Jain, V. K. \& Kumar, S. (2018), 'Rough set based intelligent approach for identification of h1n1 suspect using social media', Kuwait Journal of Science 45(2).

Javitt, D. C. (2010), 'Glutamatergic theories of schizophrenia', The Israel journal of psychiatry and related sciences 47(1), 4.

Kapur, S. \& Remington, G. (1996), 'Serotonin dopamine interaction and its relevance to schizophrenia', American Journal of Psychiatry 153(4), 466-476.

Kaskie, R. E.; Graziano, B. \& Ferrarelli, F. (2017), 'Schizophrenia and sleep disorders: links, risks, and management challenges', Nature and science of sleep 9, 227.

Kendler, K. S.; Gruenberg, A. M. \& Tsuang, M. T. (1985), 'Subtype stability in schizophrenia.', The American journal of psychiatry.

Keri, S.; Kiss, I. \& Kelemen, O. (2009), 'Sharing secrets: oxytocin and trust in schizophrenia', Social neuroscience 4(4), 287-293.

Korpi, E. R.; Kleinman, J. E.; Goodman, S. I.; Phillips, I.; DeLisi, L. E.; Linnoila, M. and Wyatt, R. J. (1986), 'Serotonin and 5hydroxyindoleacetic acid in brains of suicide victims: comparison in chronic schizophrenic patients with suicide as cause of death', Archives of General Psychiatry 43(6), 594-600.

Kraal, A. Z.; Arvanitis, N. R.; Jaeger, A. P. and Ellingrod, V. L. (2019), 'Could dietary glutamate play a role in psychiatric distress?', Neuropsychobiology.

Kumar, A. \& Chatterjee, I. (2016), 'Data mining: An experimental approach with weka on uci dataset', International journal of computer applications 138(13), 23-28. DOI:

https://dx.doi.org/10.5120/ijca201690905 $\underline{0}$

Laruelle, M.; Abi-Dargham, A.; Gil, R.; Kegeles, L. \& Innis, R. (1999), 'Increased dopamine transmission in schizophrenia: relationship to illness phases', Biological psychiatry 46(1), 56-72.

Laruelle, M.; Kegeles, L. S. \& AbiDargham, A. (2003), 'Glutamate, dopamine, and schizophrenia', Ann NY Acad Sci 1003, 138-158. 
Lewis, D. A. \& Levitt, P. (2002), 'Schizophrenia as a disorder of neurodevelopment', Annual review of neuroscience 25(1), 409-432.

Lieberman, J. A. \& Koreen, A. R. (1993), 'Neurochemistry and neuroendocrinology of schizophrenia: a selective review', Schizophrenia Bulletin 19(2), 371-429.

Lydiard, R. B. (2003), 'The role of gaba in anxiety disorders.', The Journal of clinical psychiatry 64, 21-27.

MacDonald, K. \& Feifel, D. (2012), 'Oxytocin in schizophrenia: a review of evidence for its therapeutic effects', Acta neuropsychiatrica 24(3), 130-146.

Malik, Z.; Baik, D. \& Schey, R. (2015), 'The role of cannabinoids in regulation of nausea and vomiting, and visceral pain', Current gastroenterology reports 17(2), 9 .

McCutcheon, R. A.; Abi-Dargham, A. \& Howes, O. D. (2019), 'Schizophrenia, dopamine and the striatum: from biology to symptoms', Trends in neurosciences 42(3), 205-220.

McCutcheon, R. A.; Marques, T. R. \& Howes, O. D. (2020), 'Schizophreniaan overview', JAMA psychiatry 77(2), 201-210.

Meltzer, H. Y.; Busch, D. \& Fang, V. S. (1981), 'Hormones, dopamine receptors and schizophrenia', Psychoneuroendocrinology 6(1), 17-36.
Menschikov, P.; Semenova, N.; Ublinskiy, M.; Akhadov, T.; Keshishyan, R.; Lebedeva, I.; Omelchenko, M.; Kaleda, V. and Varfolomeev, S. (2016), 1 h-mrs and mega-press pulse sequence in the study of balance of inhibitory and excitatory neurotransmitters in the human brain of ultra-high risk of schizophrenia patients, in 'Doklady Biochemistry and biophysics', Vol. 468, Springer, pp. 168-172.

Meyer, J. M. (2014), 'The glutamate hypothesis of schizophrenia', J. Clin. Psychiatry 75, e18.

Mittal, R.; Debs, L. H.; Patel, A. P.; Nguyen, D.; Patel, K.; O'Connor, G.; Grati, M.; Mittal, J.; Yan, D.; Eshraghi, A. A. et al. (2017), 'Neurotransmitters: The critical modulators regulating gutbrain axis', Journal of cellular physiology 232(9), 2359-2372.

Moghaddam, B. (2003), 'Bringing order to the glutamate chaos in schizophrenia', Neuron 40(5), 881-884.

Monti, J. M. \& Monti, D. (2004), 'Sleep in schizophrenia patients and the effects of antipsychotic drugs', Sleep medicine reviews 8(2), 133-148.

Monti, J. M. \& Monti, D. (2005), 'Sleep disturbance in schizophrenia', International Review of Psychiatry 17(4), 247-253.

Moses, D. et al. (2015), 'A survey of data mining algorithms used in cardiovascular disease diagnosis from multi-lead ecg data', Kuwait Journal of Science 42(2). 
Nicholls, J. G.; Martin, A. R.; Wallace, B. G. \& Fuchs, P. A. (2001), From neuron to brain, Vol. 271, Sinauer Associates Sunderland, MA.

Ohuoha, D. C.; Hyde, T. M. and Kleinman, J. E. (1993), 'The role of serotonin in schizophrenia: an overview of the nomenclature, distribution and alterations of serotonin receptors in the central nervous system', Psychopharmacology 112(1), S5-S15.

Owens, M. J. \& Nemeroff, C. B. (1994), 'Role of serotonin in the pathophysiology of depression: focus on the serotonin transporter.', Clinical chemistry 40(2), 288-295.

Peitl, V.; Ivanc'ic' Ravlic', I. \& Golubic' Zatezalo, V. (2020), 'Genetic polymorphisms of the dopamine and serotonin systems in schizophrenia in relation to violence and aggression', Archives of Psychiatry Research: An International Journal of Psychiatry and Related Sciences 56(1), 63-74.

Petroff, O. A.; Rothman, D. L.; Behar, K. L. \& Mattson, R. H. (1996), 'Low brain gaba level is associated with poor seizure control', Annals of Neurology: Official Journal of the American Neurological Association and the Child Neurology Society 40(6), 908-911.

Petroff, O. A. (2002), 'Book review: Gaba and glutamate in the human brain', The Neuroscientist 8(6), 562-573.
Raine, A. (2018), 'Antisocial personality as a neurodevelopmental disorder', Annual review of clinical psychology 14, 259289.

Rajkumar, N. \& Palanichamy, J. (2015), 'Optimized construction of various classification models for the diagnosis of thyroid problems in human beings', Kuwait Journal of Science 42(2).

Ressler, K. J. \& Nemeroff, C. B. (1999), 'Role of norepinephrine in the pathophysiology and treatment of mood disorders', Biological psychiatry 46(9), 1219-1233.

Reynolds, G. P. (1983), 'Increased concentrations and lateral asymmetry of amygdala dopamine in schizophrenia', Nature 305(5934), 527.

Schultz, S. H.; North, S. W.; Shields, C. G. et al. (2007), 'Schizophrenia: a review', Am Fam Physician 75(12), 1821-1829.

Seeman, P. (1980), 'Brain dopamine receptors.', Pharmacological Reviews 32(3), 229-313.

Shah, U. H. \& Gonza'lez-Maeso, J. (2019), 'Serotonin and glutamate interactions in preclinical schizophrenia models', ACS chemical neuroscience.

Snyder, S. $\quad$ H.; $\quad$ Banerjee, S. P.; Yamamura, H. I. \& Greenberg, D. (1974), 'Drugs, neurotransmitters, and schizophrenia', Science 184(4143), 12431253. 
Sternbach, H. (1991), 'The serotonin syndrome', Am J Psychiatry 148(6), 705713.

Stone, J. M.; Morrison, P. D.; \& Pilowsky, L. S. (2007), 'Glutamate and dopamine dysregulation in schizophrenia a synthesis and selective review', Journal of psychopharmacology 21(4), 440-452.

Swanton, T. (2020), 'The dopamine, glutamate, and gaba hypotheses of schizophrenia: Glutamate may be the key', ANU Undergraduate Research Journal 10(1), 88-96.

Tang, W.; Yao, X. \& Zheng, Z. (1994), 'Rehabilitative effect of music therapy for residual schizophrenia: A one-month randomised controlled trial in shanghai', The British journal of psychiatry 165(S24), 38-44.

Toru, M.; Watanabe, S.; Shibuya, H.; Nishikawa, T.; Noda, K.; Mitsushio, H.; Ichikawa, H.; Kurumaji, A.; Takashima, M.; Mataga, N. et al. (1988), 'Neurotransmitters, receptors and neuropeptides in post-mortem brains of chronic schizophrenic patients', Acta Psychiatrica Scandinavica 78(2), 121137.

Tost, H.; Alam, T. \&MeyerLindenberg, A. (2010), 'Dopamine and psychosis: theory, pathomechanisms and intermediate phenotypes', Neuroscience \& Biobehavioral Reviews 34(5), 689-700.

Tsai, G. \& Coyle, J. T. (2002), 'Glutamatergic mechanisms in schizophrenia', Annual review of pharmacology and toxicology 42(1), 165179.

Wassef, A.; Baker, J. \& Kochan, L. D. (2003), 'Gaba and schizophrenia: a review of basic science and clinical studies', Journal of clinical psychopharmacology 23(6), 601-640.

Watanabe, M.; Maemura, K.; Kanbara, K.; Tamayama, T. \& Hayasaki, H. (2002), Gaba and gaba receptors in the central nervous system and other organs, in 'International review of cytology', Vol. 213, Elsevier, pp. 1-47.

Weinberger, D. R. (1987), 'Implications of normal brain development for the pathogenesis of schizophrenia', Archives of general psychiatry 44(7), 660-669.

Werner, F.-M. \& Coven as, R. (2013), 'Classical neurotransmitters and neuropeptides involved in schizophrenia: how to choose the appropriate antipsychotic drug?', Current Drug Therapy 8(2), 132-143.

Wise, R. A. (2004), 'Dopamine, learning and motivation', Nature reviews neuroscience 5(6), 483.

Zhou, Y.; Liang, M.; Tian, L.; Wang, K.; Hao, Y.; Liu, H.; Liu, Z. and Jiang, T. (2007), 'Functional disintegration in paranoid schizophrenia using resting-state fmri', Schizophrenia research 97(1-3), 194-205.

Zhou, Y. \& Danbolt, N. (2014), 'Glutamate as a neurotransmitter in the 
healthy brain', Journal of neural transmission 121(8), 799-817.

Submitted : $27 / 02 / 2020$

Revised : 26/05/2020

Accepted : 30/06/2020

DOI $\quad: 10.48129 /$ kjs.v48i2.9264 